\title{
IDENTIFICATION OF SPONTANEOUS SPOKEN TEXTS IN SLOVAK
}

\author{
RÓBERT SABO - PETER KRAMMER - JÁN MOJŽIŠ - MARCEL KVASSAY \\ Institute of Informatics, Slovak Academy of Sciences, Bratislava, Slovakia
}

SABO, Róbert - KRAMMER, Peter - MOJŽIŠ, Ján - KVASSAY, Marcel: Identification of spontaneous spoken texts in Slovak. Journal of Linguistics, 2019, Vol. 70, No 2, pp. $481-490$.

\begin{abstract}
We propose a text classification method for the purpose of creating a language model for automatic recognition of spontaneous spoken speech. Transcripts from our departmental speech database served as spontaneous spoken texts. Using supervised machine learning methods, we have created multiple classification models (including neural networks), that were able to distinguish them from written texts with high accuracy. We subsequently verified the accuracy of our trained models on a database of texts containing direct speech extracted from newspaper articles.

Keywords: spontaneous speech, text classification, supervised machine learning, neural networks, Slovak language
\end{abstract}

\section{INTRODUCTION}

The automatic speech recognition technology is currently used in various areas of life. A few years ago, it was used mostly in justice, medicine and automated dialogue systems where the limited domain and established rules of text creation enabled it to achieve high recognition accuracy. Nowadays, thanks to the use of neural networks, automatic speech recognition is introduced into areas where spontaneous speech is used. Automatic recognition of spontaneous speech requires special approaches not only to the acoustic modeling but also to the language modeling [1]. In this paper we focus on the acquisition of text material for training a language model for automatic recognition of spontaneous speech.

In text classification, researchers mostly focus on classification by content, theme, genre, and so on. The use of classification methods to distinguish the style or the form of text (spoken versus written) is less common.

In Slovak, only text categorization techniques (latent Dirichlet allocation) were used in an article by D. Zlacky et al. [2], which led to an increase in accuracy. However, this method is not suitable for our purpose.

In principle, we could use approaches based on human-designed features [3] or those without them [4]. We have opted for defining our own features because we made assumptions about important characteristics of our texts but did not have sufficiently representative data for training the classifier. 
The aim of our study is not to create a better language model for the particular task of automatic recognition of spontaneous speech, but to articulate a choice of suitable methods for classifying spontaneous spoken texts and to reveal the typical features of spoken text that could help to identify it.

\section{TEXTS ACQUISITION}

For our purposes (i.e., creating language models) we have chosen the texts from our departmental text database.

\subsection{Spontaneous spoken texts acquisition}

For the purpose of creating a model we used a language model for spontaneous speech recognition we have chosen the annotations (annotated transcripts) from our departmental speech database as the spontaneous texts. Their number is quite limited, but other available texts, such as movie subtitles or direct speech extracted from written texts are somehow modified and therefore not fully authentic.

Specifically, we have chosen the annotations of interviews from the portal "100názorov" [5]. The portal collects short interviews (about 10 minutes each) with personalities of cultural and social life on various topics. We created two databases: the database "100n_all" with all the interviews and the database "100n_polit" only with the interviews on the subject of politics.

Special annotation labels for various acoustic events (background noise, hesitations, breaths) were removed from texts but punctuation was left intact for further processing. All annotated segments were linked together and then divided into rows (records), each row containing 160 words, corresponding to the average article length of the "Nový Čas" journal, which was our source of written texts (see next subsection). As a result, the database "100n_all" consisted of 2575 lines (representing 270 interviews) and the database "100n_polit" of 315 lines (33 interviews).

\subsection{Written texts acquisition}

Written texts were obtained using a standard web crawling technique focusing on the Cas.sk news portal (domestic politics category) [6] as our data source. We installed Web Scraper (https://www.webscraper.io/) in our Chrome browser, created a template based on the Cas.sk structure and then extracted the article texts. A total of 7702 articles were obtained, dated between 2019/02/13 and 2013/12/12 (inclusive). The articles contained 160 words on average. Like our spoken texts, these articles have been linked to create a file containing 7702 lines. Both files (spoken texts and news texts) were further preprocessed as detailed in the next section. 


\section{TEXT PREPROCESSING}

Preprocessing was dependent on the type of text. There were 7 different preprocessing steps: 1. surnames removal, 2. abbreviations and numbers removal, 3 . names removal, 4. dots, commas and lone letters removal, 5. extra spaces removal, 6. lowercase conversion, 7. lemmatization. For written news article texts, there was one extra preprocessing step: since Web Scraper stores each article in fragments (based on template), these fragments had to be linked together in the right order.

Surnames removal is a dictionary filter containing the surnames of well-known people (e.g. politicians). Abbreviations and number removal was also a dictionary filter, populated with well-known universities and organizations (web addresses were also removed). Names removal filtered out all words starting with a capital letter but not placed at the beginning of a sentence. This preprocessing sometimes produced residual isolated letters with no semantic meaning. Dots, commas and lone letters filter removed all these. Moreover, extra spaces may have been generated by each previous filter that replaced unwanted words with spaces in order to prevent artificial joining of the remaining words and letters. The removal of extra spaces was followed by a lowercase letter conversion and by lemmatization, respectively. Some of these filters relied on regular expressions (like names or spaces removals).

Lemmatization was performed using the online service "Morfologická dezambiguácia" [7]. The service is based on the open source MorphoDiTa tool [8], which combines a tagger, an entity recognizer, and a text analyzer. A local MorphoDiTa client was created and queried via HTTP POST requests.

"Morfologická dezambiguácia" service also provided text analysis, which we have used to obtain additional information about the relevant verbs, such as their grammatical person and number.

\section{METHOD}

\subsection{Selection of typical ngrams}

Since our goal was to identify spontaneous spoken texts, we used aggregated word frequency statistics from the corpus "Hovor" [9] to define our classification attributes. This corpus contains 6,5 million words (tokens) from different areas and can serve as a representative corpus of Slovak spoken speech.

We used the most common unigrams, bigrams and trigrams from this corpus as candidate indicators of the "spokeness" of text. For each row (record) in our datasets, we calculated how many of its n-grams occurred in the "Hovor" corpus and what was their frequency there (this tells us how typical the n-gram is for spoken speech). In order to avoid potential overfitting of our models through topic-related words, we only used unigrams with corpus frequency of at least 719 (which represent the 600 most frequent words), as well as bigrams with at least 30 occurrences and trigrams 
with at least 11 occurrences in the corpus. In what follows we refer to these three categories as "the frequent spoken n-grams" in Slovak.

\subsection{Classification models}

\section{Experiment 1}

The training of our classification models was realized in Weka [10]. We used primarily Radial Basis Functions (RBF), Multi Layer Perceptron (MLP), Support Vector Machine (SVM SMO), Random Forest, and Linear Discriminant Analysis (LIDA), with various parameters and settings. We then used F-measure Score (F1 Score) and Area Under Curve Receiver Operating Characteristic (AUC ROC) as our model accuracy criterion. Finally, 20-fold cross validation was used in order to obtain sufficiently objective accuracy estimates.

In the first phase, classification models were created based only on the following 12 numerical attributes:

- $\quad$ the first 3 attributes (per1sg, per2sg, per3sg) contained the counts (frequencies) of verbs in the singular of the $1^{\text {st }}, 2^{\text {nd }}$ and $3^{\text {rd }}$ grammatical person, respectively, in each record;

- $\quad$ the next 3 attributes (per1pl, per2pl, per3pl) contained the corresponding plural counts;

- $\quad$ further 3 attributes (1-gram, 2-gram, 3-gram) contained aggregated n-gram frequencies $(\mathrm{n}=1,2,3)$ in each record for the frequent spoken n-grams;

and the last 3 attributes (1-count, 2-count, 3-count) contained weighted sums of n-gram frequencies $(\mathrm{n}=1,2,3)$ in each record for "the frequent spoken" $\mathrm{n}$-grams, with their frequencies in the corpus serving as weights. In order to make all these attributes mutually comparable, we standardized them.

Our trained models achieved the accuracies listed in Tab. 1. The significance of each input attribute (feature) is shown in Tab. 2, expressed through several alternative metrics used in [12], such as Information Gain (InfoGain), Gain Ratio [11] (GainRatio), Correlation Coefficient (Correl), Chi Square [11] (Chi2), and Signification Evaluation [12] (SignEval). Overall, the most significant attribute was (unsurprisingly) the indicator of the grammatical first person (singular) ("per1sg"), followed by its plural counterpart ("perlpl") and the count of the frequent spoken bigrams ("2-count"). For more details, see the "Discussion" section.

\begin{tabular}{|l|c|c|}
\hline \multicolumn{1}{|c|}{ Model Type } & F1 Score & AUC ROC \\
\hline MLP Classifier & 0,987 & 0,998 \\
\hline Random Forest & 0,983 & 0,997 \\
\hline RBF Classifier & 0,965 & 0,991 \\
\hline Voted Perceptron & 0,956 & 0,936 \\
\hline SVM SMO & 0,947 & 0,919 \\
\hline LIDA Classifier & 0,934 & 0,984 \\
\hline
\end{tabular}

Tab. 1. Comparison of performance for models using the 12 input attributes 


\begin{tabular}{|c|c|c|c|c|c|}
\hline Attribute & InfoGain & GainRatio & Chi2 & SignEval & Correl \\
\hline per1sg & 0,5016 & 0,3952 & 7174,570 & 0,755 & 0,6462 \\
\hline per2sg & 0,0261 & 0,0927 & 426,434 & 0,406 & 0,1844 \\
\hline per3sg & 0,0551 & 0,0239 & 670,714 & 0,195 & 0,1892 \\
\hline per1pl & 0,2677 & 0,2240 & 4105,102 & 0,655 & 0,5335 \\
\hline per2pl & 0,0127 & 0,0376 & 205,771 & 0,195 & 0,0846 \\
\hline per3pl & 0,0158 & 0,0101 & 238,122 & 0,126 & 0,1484 \\
\hline 1-gram & 0,4012 & 0,1529 & 4997,830 & 0,495 & 0,2130 \\
\hline 2-gram & 0,4085 & 0,1360 & 5312,871 & 0,500 & 0,4095 \\
\hline 3-gram & 0,1433 & 0,1148 & 2208,187 & 0,378 & 0,4022 \\
\hline 1-count & 0,3884 & 0,1554 & 4846,620 & 0,475 & 0,3110 \\
\hline 2-count & 0,4907 & 0,1979 & 6598,433 & 0,561 & 0,6217 \\
\hline 3-count & 0,1431 & 0,0821 & 2193,327 & 0,382 & 0,3599 \\
\hline
\end{tabular}

Tab. 2. Attribute importance metrics for our 12 numerical features

In the next phase, we tried an alternative approach based on the bag-of-words representation of the texts themselves, which we repeated twice: with and without lemmatization. In Slovak, lemmatization has a profound effect on the size of the vocabulary. In our case the vocabulary was reduced by more than $60 \%$ (from 87838 words to 34023). Even after this reduction it was clear, however, that not all words would be significant in distinguishing the two classes. Therefore, we used Principal Component Analysis (PCA [13]) to identify and extract 200 most significant components (linear combinations of individual word representations).

We then trained several different classifiers on these principal components as input attributes. Tables 3 and 4 show the accuracies achieved (with and without lemmatization, respectively). Again, 20-fold cross-validation was used.

\begin{tabular}{|l|c|c|}
\hline \multicolumn{1}{|c|}{ Model Type } & F1 Score & AUC ROC \\
\hline MLP Classifier & 0,996 & 1,000 \\
\hline LIDA Classifier & 0,995 & 1,000 \\
\hline SVM SMO & 0,996 & 0,995 \\
\hline RBF Classifier & 0,987 & 0,998 \\
\hline Random Forest & 0,981 & 0,998 \\
\hline Voted Perceptron & 0,993 & 0,993 \\
\hline $\begin{array}{l}\text { SVM SMO + RBF } \\
\text { Kernel }\end{array}$ & 0,991 & 0,989 \\
\hline
\end{tabular}

Tab. 3. Comparison of classification model performance with lemmatization

\begin{tabular}{|l|c|c|}
\hline \multicolumn{1}{|c|}{ Model Type } & F1 Score & AUC ROC \\
\hline MLP Classifier & 0,996 & 1,000 \\
\hline Voted Perceptron & 0,991 & 0,989 \\
\hline
\end{tabular}




\begin{tabular}{|l|c|c|}
\hline \multicolumn{1}{|c|}{ Model Type } & F1 Score & AUC ROC \\
\hline LIDA Classifier & 0,985 & 0,997 \\
\hline SVM SMO & 0,988 & 0,981 \\
\hline Random Forest & 0,976 & 0,997 \\
\hline RBF Classifier & 0,937 & 0,965 \\
\hline
\end{tabular}

Tab. 4. Comparison of classification model performance without lemmatization

From the models we have trained so far, we can summarize the following best settings for each model type:

- $\quad$ RBF Classifier: Number of RBF functions $=8$, Tolerance $=1.0 \mathrm{e}-6$, Number of decimal places $=6$, using Conjugate Gradient Descent, without using Normalized Basis Functions

- $\quad$ MLP Classifier: Number of hidden units $=8$, Ridge $=0.01$, Activation Function = Approximate Sigmoid

- $\quad$ SVM SMO: Complexity Parameter $=1.0$, epsilon for Round off error $=1.0 \mathrm{e}-$ 12, Tolerance parameter $=0.001$

- $\quad$ Random Forest: Number of iteration $=100$, maximal depth $=$ unlimited

- $\quad$ LIDA Classifier: Number of decimal places $=6$, Ridge $=1.0 \mathrm{e}-6$

\section{Experiment 2}

The results from Experiment 1 (AUC ROC and F1 Score) are definitely encouraging, given that they were achieved in a rather demanding setting, since only political articles were included in Class 1, but a mixture of topics in Class 0 . Subsequently, we decided to test the robustness of our models by testing them on data from a different source.

Therefore, in this second experiment, we created a test set with 19631 records. Of these, 16929 records came from a new (SITA) data source that contained direct speech (class 0). Next 2720 test set records were added from Cas.sk (written texts belonging to class 1 ). As in the $1^{\text {st }}$ experiment, the training set consisted of data from the 100n and Cas.sk datasets (with the number of records from Cas.sk reduced to 5000). Creation of an independent test set allowed us to perform Hold-Out validation in addition to cross validation.

The same model types as in experiment 1 were used for classification on the basis of the 12 attributes listed in Table 2. Validation results for individual models are shown in Table 5.

\begin{tabular}{|c|c|c|c|c|c|c|}
\hline & \multicolumn{2}{|c|}{ 20 Fold Cross Validation } & \multicolumn{4}{c|}{ Hold Out Validation } \\
\hline Model Type & F1 Score & AUC & F1 Score & AUC & Precision & Recall \\
\hline MLP Classifier & 0,974 & 0,992 & 0,956 & 0,987 & 0,961 & 0,954 \\
\hline
\end{tabular}




\begin{tabular}{|c|c|c|c|c|c|c|}
\hline & \multicolumn{2}{|c|}{ 20 Fold Cross Validation } & \multicolumn{4}{|c|}{ Hold Out Validation } \\
\hline Model Type & F1 Score & AUC & F1 Score & AUC & Precision & Recall \\
\hline RBF Classifier & 0,970 & 0,994 & 0,934 & 0,987 & 0,950 & 0,929 \\
\hline Random Forest & 0,981 & 0,998 & 0,918 & 0,988 & 0,943 & 0,909 \\
\hline SVM - SMO & 0,942 & 0,930 & 0,908 & 0,928 & 0,937 & 0,898 \\
\hline LIDA Classifier & 0,925 & 0,983 & 0,840 & 0,976 & 0,918 & 0,814 \\
\hline
\end{tabular}

Tab. 5. Comparison of classification model performance

\section{DISCUSSION}

Since our goal in this paper was to identify the style rather than topic, in the preprocessing stage we have removed from our texts all proper names and abbreviations, which carry primarily content-related information. The methods chosen for classification did not take into account the relationship between words (context), thus affecting the semantics (meaning), but in our case, this was an advantage.

In consequence, already in the first experiment, we have achieved encouraging classification results. Using just the 12 numerical input attributes to characterize our input data greatly reduced the computational complexity of our models, and yet the F1 scores of the two best ones (MLP Classifier and Random Forest) surpassed 0.98. Overall, the most influential attributes contributing to this result were the two frequency indicators of the grammatical first person in verbs (singular and plural), followed by the attributes derived from the counts of the frequent spoken unigrams and bigrams.

The significance of each input attribute is listed in Table 2, which also shows relatively lower significance for the indicators of the grammatical $2^{\text {nd }}$ and $3^{\text {rd }}$ person (especially in plural).

Regarding the significance of attributes derived from the frequent spoken n-gram counts, we can see that for unigrams and 3 -grams, the non-weighted attributes (1-gram, 3-gram) reach significances similar to their weighted counterparts (1-count, 3-count). Somewhat surprisingly, for bigrams, the 2-count weighted attribute consistently outperformed its non-weighted counterpart (2-gram) across all the monitored significance criteria.

For the classification based on the bag-of-words models, slightly better results were achieved (Tables 3 and 4). The best models achieved the F1 score above 0.99 (both with and without lemmatization). In this case, we used 200 most important components from PCA analysis. Lemmatization reduced the dictionary from 87838 to 34023 words. 
By clubbing together all the forms of a given word, lemmatization allows its frequency to be estimated more objectively. On the downside, it also removes the signals of the grammatical person which appeared to be significant in our previous models. However, the latter loss is more than outweighed by other signals of spoken speech that still enabled our "lemmatized" classifiers to perform very well.

An important advantage of the approach based on the 12 numerical attributes is that it achieved similar classification performance as the bag-of-words approach, which required lengthy and complex PCA analysis. Its second advantage is the ease of determining the significance of each attribute.

Of course if we process only one dataset, it could be argued that it would take longer to define those 12 numerical attributes than to blindly run the 200-principal component model without trying to interpret its components. But if we consider that more datasets could be processed through our 12 numerical attributes, their benefits would then multiply.

In all the cases discussed so far, the best results were achieved by the MLP Classifier, while the Random Forest model produced solid results too. Surprisingly, the RBF Classifier achieved somewhat weaker results; in some cases it was even worse than Random Forest.

In the second experiment with SITA data in the test set, there was a more pronounced decline in F1 scores. This was due to the different type of the "spoken" SITA texts (quoted direct speech) than those in the training set (transcripts of spontaneous spoken speech). Table 5 shows this decrease for Hold-out validation with the SITA data. The accuracy is still quite high, however. To identify spontaneous spoken texts in a larger corpus, it is important to have high accuracy. As evident from Table 5 for Hold-Out validation, precision is higher than recall for each method. Overall, the MLP Classifier was the most accurate. Although it was outperformed by 2 models (RBF Classifier, Random Forest) in cross validation, it was the top performer in the more challenging Hold-Out validation.

Overall, the distinctive suitability of the MLP model for this type of task is clear. Another interesting aspect is the way in which it is possible to distinguish spontaneous spoken texts in Slovak. With the 12 numerical input attributes (summarizing the information about the grammatical person, number and n-gram counts) the dominant factor is the first person information obtained from the verbs (a characteristic typical of the Slovak language). In lemmatized text, the grammatical person information is lost as the verb is modified to its basic form. However, that information can still be obtained from personal pronouns remaining in the text. However, the high number of different word forms results in a more extensive dictionary as well as lower frequencies (and thus lower representativeness) of the training set, which could be problematic in some contexts. 


\section{CONCLUSIONS AND FUTURE WORK}

This paper dealt with the distinction between spontaneous spoken and written texts in the Slovak language. In the process, a number of aspects (grammatical person, personal pronouns as well as typical n-grams for spontaneous speech) were revealed as relevant for successful classification. An important factor was the influence of grammatical person, which can be identified from personal pronouns, but in Slovak also from verbs. Therefore, in three distinct cases (lemmatized text, non-lemmatized text, and classification based on 12 numerical attributes), significantly similar results were achieved, with relatively high success. An important advantage of the approach with the 12 numerical attributes is the considerably faster training and classification compared to the 200-principal component model. Its second advantage is the easy determination of the significance of each attribute (since its attributes are not transformed by the PCA).

In our experiments, the MLP classifier achieved the highest accuracy in most cases, which indicated its preferability for this type of classification. Noteworthy results were also achieved by the Random Forest model, given its simplicity and speed of training. Both types of models achieved remarkable F1 scores between 0.97 and 0.99 (verified by 20-fold cross-validation). Their validation on another Hold-Out dataset of a slightly different character (SITA), reduced their accuracy somewhat, but their F1 scores, precision, and recall still remained above 0.95 , which we consider a very good result.

As an alternative, we would also consider comparing our approach with pretrained models such as word2vec or fasttext.

As the next step we plan to create a specialized language model from the texts we have classified as spoken and evaluate the accuracy of automatic speech recognition for spontaneous spoken speech.

In the future, we also plan to use the classification to differentiate the text topic (e.g. sport, politics, religion, etc.). Subsequently, we will distinguish whether the text is written or spoken for each topic separately, which, we hope, will help us develop even more accurate language models for spontaneous speech recognition.

\section{ACKNOWLEDGMENTS}

The research leading to the results presented in this paper was supported by grants VEGA 2/0161/18, VEGA 2/0155/19, and U-COMP APVV-17-0619.

\section{References}

[1] Chou, W., and Juang, B. H. (Eds.). (2003). Pattern recognition in speech and language processing. CRC Press. 
[2] Zlacký, D., Staš, J., Juhár, J., and Čižmár, A. (2014). Text categorization with latent Dirichlet allocation. Journal of electrical and electronics engineering 7(1), pages 161-164.

[3] Haddoud, M., Mokhtari, A., Lecroq, T., and Abdeddaïm, S. (2016). Combining supervised term-weighting metrics for SVM text classification with extended term representation. Knowledge and Information Systems, 49(3), pages 909-931.

[4] Lai, S., Xu, L., Liu, K., and Zhao, J. (2015). Recurrent convolutional neural networks for text classification. In Twenty-ninth AAAI conference on artificial intelligence.

[5] 100 názorov. Accessible at: http://100nazorov.sk/

[6] Politika. Nový čas. FPD Media. Accessible at: https://www.cas.sk/spravy/ politika

[7] Garabík, R.: Morfologická dezambiguácia. Accessible at: https://morphodita.juls. savba.sk/

[8] Straková, J., Straka, M., and Hajič, J. (2014). Open-source tools for morphology, lemmatization, POS tagging and named entity recognition. In Proceedings of $52^{\text {nd }}$ Annual Meeting of the Association for Computational Linguistics: System Demonstrations, pages 13-18.

[9] Slovenský hovorený korpus - s-hovor-6.0. Bratislava, Jazykovedný ústav L'. Štúra SAV 2017. Accessible at: http: / / korpus.juls.savba.sk

[10] Hall, M., Frank, E., Holmes, G., Pfahringer, B. Reutemann, P. and Witten, I. H. (2016). The WEKA Data Mining Software: An Update. SIGKDD Explorations, 11(1), 2009. E. Frank, M. A. Hall, and I. H. Witten: Data Mining: Practical Machine Learning Tools and Techniques, Morgan Kaufmann, Fourth Edition.

[11] Novaković, J., Strbac, P., and Bulatović, D. (2011). Toward Optimal Feature Selection Using Ranking Methods and Classification Algorithms, Yugoslav Journal of Operations Research 21, pages 119-135.

[12] Ahmad, A., and Dey, L. (2004). A feature selection technique for classificatory analysis. Accessible at: http://citeseerx.ist.psu.edu/viewdoc/download?doi=10. $1.1 .520 .6722 \& r e p=r e p 1 \& t y p e=p d f$

[13] Guan, Y., and Dy, J. (2009). Sparse Probabilistic Principal Component Analysis. Accessible at http://proceedings.mlr.press/v5/yue09a/yue09a.pdf 